\title{
Regulation of mitochondrial permeability transition pore by PINK1
}

Clement A Gautier ${ }^{1,4 \dagger}$, Emilie Giaime ${ }^{1 \dagger}$, Erica Caballero², Lucía Núñez ${ }^{2}$, Zhiyin Song $^{3}$, David Chan ${ }^{3}$, Carlos Villalobos ${ }^{2}$ and Jie Shen ${ }^{1 *}$

\begin{abstract}
Background: Loss-of-function mutations in PTEN-induced kinase 1 (PINK1) have been linked to familial Parkinson's disease, but the underlying pathogenic mechanism remains unclear. We previously reported that loss of PINK1 impairs mitochondrial respiratory activity in mouse brains.

Results: In this study, we investigate how loss of PINK1 impairs mitochondrial respiration using cultured primary fibroblasts and neurons. We found that intact mitochondria in PINK1-/- cells recapitulate the respiratory defect in isolated mitochondria from PINK1-/- mouse brains, suggesting that these PINK1-/- cells are a valid experimental system to study the underlying mechanisms. Enzymatic activities of the electron transport system complexes are normal in PINK1-/- cells, but mitochondrial transmembrane potential is reduced. Interestingly, the opening of the mitochondrial permeability transition pore (MPTP) is increased in PINK1-/- cells, and this genotypic difference between PINK1-/- and control cells is eliminated by agonists or inhibitors of the mPTP. Furthermore, inhibition of mPTP opening rescues the defects in transmembrane potential and respiration in PINK1-/- cells. Consistent with our earlier findings in mouse brains, mitochondrial morphology is similar between PINK1-/- and wild-type cells, indicating that the observed mitochondrial functional defects are not due to morphological changes. Following FCCP treatment, calcium increases in the cytosol are higher in PINK1-/- compared to wild-type cells, suggesting that intra-mitochondrial calcium concentration is higher in the absence of PINK1.
\end{abstract}

Conclusions: Our findings show that loss of PINK1 causes selective increases in MPTP opening and mitochondrial calcium, and that the excessive mPTP opening may underlie the mitochondrial functional defects observed in PINK1 $-/-$ cells.

Keywords: Parkinson's disease, Mitochondrial respiration, Mitochondrial transmembrane potential, Mitochondrial permeability transition pore, Calcium

\section{Introduction}

Recessively inherited loss of function mutations in the PINK1 gene have been linked to familial Parkinson's disease (PD). The PINK1 protein bears a 34 amino acid mitochondrial targeting domain [1] and has been shown to localize within mitochondria [2]. Mitochondrial dysfunction has long been thought to play a key role in PD pathogenesis, based in part on postmortem studies that

\footnotetext{
* Correspondence: jshen@rics.bwh.harvard.edu

${ }^{+}$Equal contributors

${ }^{1}$ Center for Neurologic Diseases, Department of Neurology, Brigham \& Women's Hospital, Program in Neuroscience, Harvard Medical School, Boston, MA 02115, USA

Full list of author information is available at the end of the article
}

showed mitochondrial impairment (e.g. reduced complex I activity) and oxidative damage in idiopathic PD brains [3]. This is further supported by observations that mitochondrial complex I inhibitors, such as MPTP [4] and rotenone [5] produce parkinsonian syndromes in humans and experimental animal models.

Genetic studies in Drosophila showed that PINK1 is involved in the maintenance of mitochondrial morphology by interacting with components of the mitochondrial fission and fusion machinery [6-9]. Loss of PINK1 in Drosophila appears to promote mitochondrial fusion, though the effects of PINK1 inactivation on mitochondrial morphology in cultured mammalian cells are less consistent, ranging from promotion of mitochondrial fragmentation or fusion to no effects [10-14]. Despite

\section{Biomed Central}


the controversial findings on the effects of PINK1 inactivation on mitochondrial morphology in mammalian culture systems, several functional defects have been reported consistently, including impairment of mitochondrial respiration [15-20] and reduction of mitochondrial transmembrane potential $[1,11,15,16,21]$. Our previous analysis of PINK1-/- mice led to the first report showing that PINK1 is required for mitochondrial respiratory function in vivo [18]. However the cause of these functional defects remains to be elucidated.

To determine the pathogenic cascade of events in intact mitochondria, we derived primary mouse embryonic fibroblasts (MEFs) and cortical neuronal cultures from our PINK1-/- mice. Similarly to what we previously reported in isolated mitochondria from the brain, mitochondrial respiration is impaired in PINK1-/- cells. While the enzymatic activity of each complex composing the electron transport system is normal, mitochondrial transmembrane potential $\left(\Delta \Psi_{\mathrm{m}}\right)$ is reduced in PINK1-/MEFs and neurons. Interestingly, the reduction of $\Delta \Psi_{\mathrm{m}}$ in PINK1-/- cells is associated with increased opening of the mitochondrial permeability transition pore (mPTP). Inhibition of the mPTP reverses the depolarization of the mitochondrial inner membrane and respiration defects seen in PINK1-/- cells. We did not find evidences of increased oxidative stress, a common inducer of mPTP opening. In addition, we found no detectable changes in mitochondrial morphology in PINK1-/- cells. Together our findings highlight a role of PINK1 in the regulation of the mitochondrial permeability transition pore and suggest that increased opening of the pore in the absence of PINK1 may be responsible for the reduced mitochondrial transmembrane potential and the reduced respiratory activities.

\section{Materials and methods Primary MEF and cortical cultures}

Mouse embryonic fibroblasts (MEFs) were derived from embryos at embryonic day 14.5. After removing the head and the inner organs embryos were individually minced with scissors, treated with trypsin $(1 \% \mathrm{v} / \mathrm{v})$ for twelve minutes at $37^{\circ} \mathrm{C}$ and dispersed mechanically and plated with MEF media (Dulbecco's Modified Eagle Medium (DMEM), 10\% fetal bovine serum (FBS), penicillin/ streptomycin (Gibco, Life Technologies, Grand Island, NY, USA)). After they reached $\sim 100 \%$ confluency, cells were frozen down in DMEM containing 10\% DMSO (Sigma, St Louis, MO, USA). The number of MEF samples used in each experiment is specified in the figure legends and reflects the number of individual cultures derived from individual embryos used to derive MEFs.

Primary cortical cultures were prepared and maintained as described previously [22]. Experiments were performed at $14 \pm 1$ days in vitro. Cortices from different pups were not pooled, and the number of experiments specified in the legend reflects the number of different cultures derived from individual pups.

\section{Mitochondrial respiration}

Mitochondrial respiration was assayed as the $\mathrm{O}_{2}$ consumption of cell suspension using a Clark electrode (Rank Brothers Ltd, Cambridge, England). Cells were resuspended to a final density of $2.10^{6}$ cells $/ \mathrm{ml}$ in respiration buffer $(0.137 \mathrm{M} \mathrm{NaCl}, 5 \mathrm{mM} \mathrm{KCl}, 0.7 \mathrm{mM}$ $\mathrm{NaH}_{2} \mathrm{PO}_{4}, 25 \mathrm{mM}$ Tris, $\mathrm{pH} 7.4$ at $25^{\circ} \mathrm{C}$ ). Endogenous respiration activity was measured after addition of glucose $(10 \mathrm{mM}$, Sigma). For complex driven respiration, plasma membranes were permeabilized by addition of digitonin at a final concentration of $0.01 \%$ (Sigma). Cells were supplemented with substrates for either complex I (10 mM glutamate/malate, Sigma), II (10 mM succinate, Sigma) or III (1 mM TMPD/ $1 \mathrm{mM}$ ascorbate, Sigma) together with adenosine diphosphate (ADP, $1 \mathrm{mM}$, Sigma) to the recording chamber. State 3 respiration activity was then measured. ADP independent respiration activity (State 4) was monitored after addition of oligomycin ( $2 \mu \mathrm{M}$, Sigma).

\section{Enzymatic activity of ETS complexes and ATP synthase}

All assays were performed on mitochondria isolated from MEFs according to a previously established method [23]. For each complex $5 \mu \mathrm{g}$ of mitochondrial proteins and $100 \mu \mathrm{l}$ of each assay buffer were used. Complex I (NADH: ubiquinone oxidoreductase) buffer (35 mM $\mathrm{NaH}_{2} \mathrm{PO}_{4} \mathrm{pH} 7.2,5 \mathrm{mM} \mathrm{MgCl}, 0.25 \%$ BSA, $2 \mathrm{mM}$ $\mathrm{KCN}, 1 \mu \mathrm{M}$ antimycin, $97.5 \mu \mathrm{M}$ ubiquinone-1, $0.13 \mathrm{mM}$ $\mathrm{NADH}$, Sigma). Only the rotenone sensitive activity was monitored by following the oxidation of $\mathrm{NADH}$ at $340 \mathrm{~nm}\left(\mathrm{OD} 6220 \mathrm{M}^{-1} \cdot \mathrm{cm}^{-1}\right)$. Complex II (succinate dehydrogenase) buffer ( $25 \mathrm{mM} \mathrm{KH_{2 } \mathrm { PO } _ { 4 } , 5 \mathrm { mM } \mathrm { MgCl }}$, pH 7.2, $20 \mathrm{mM}$ succinate, $50 \mu \mathrm{M}$ 2,6-dichlorophenolindophenol (DCPIP), $0.25 \%$ BSA, $2 \mathrm{mM} \mathrm{KCN}, 1 \mu \mathrm{M}$ antimycin, Sigma). Enzymatic activity was monitored by the reduction of DCPIP/PES at $600 \mathrm{~nm}\left(\mathrm{OD} 19100 \mathrm{M}^{-1} . \mathrm{cm}^{-}\right.$ $\left.{ }^{1}\right)$ after addition of $65 \mu \mathrm{M}$ ubiquinone 1 . Complex III activity (decylubiquinol/ferricytochrome $\mathrm{C}$ oxidoreductase) buffer ( $3 \mathrm{mM}$ sodium azide, $1.5 \mu \mathrm{M}$ rotenone, $50 \mu \mathrm{M}$ ferricytochrome $\mathrm{C}$, and $50 \mathrm{mM}$ phosphate buffer, $\mathrm{pH}$ 7.2, Sigma). Reaction was followed as the increase in reduced Cytochrome $\mathrm{C}$ absorbance at $550 \mathrm{~nm}$ (OD $18500 \mathrm{M}^{-1} \cdot \mathrm{cm}^{-1}$ ) after the addition of $35 \mu \mathrm{M}$ of freshly prepared ubiquinol 2. Complex IV (Cytochrome C oxidase) activity and Complex II + III (succinate-Cytochrome $\mathrm{C}$ reductase) activities were previously described [18]. Levels of Cytochrome $C$ were measured by western blot using a commercial antibody (Cell signalling Technology, Danvers, MA, USA). 


\section{Measurement of mitochondrial transmembrane potential and $\mathrm{mPTP}$ opening}

Mitochondrial $\Delta \Psi$ was measured with the non-quenching Tetramethylrhodamine, methyl ester (TMRM) fluorescence methods (Molecular Probes, Life Technologies). MEFs were stained with TMRM (50 nM) in DMEM for $30 \mathrm{~min}$ at $37^{\circ} \mathrm{C}$ in the dark. The cells were then washed twice with PBS. Mitochondrial PTP opening was assessed by the quenching of calcein-AM fluorescence by cobalt [24]. Thirty min after cells were loaded with Calcein-AM (1 $\mathrm{MM}$, Molecular Probes, Life Technologies) at $37^{\circ} \mathrm{C}$ in the dark, $\mathrm{CoCl}_{2}$ (1 mM, Sigma) was added and cells incubated for another $10 \mathrm{~min}$. Then, fluorescence of 30,000 cells for each experiment was measured with a flow cytometer (FACSCalibur), and the data were processed with the CellQuest program (BD Biosciences, San Jose, CA, USA). Neurons were incubated for $30 \mathrm{~min}$ with TMRM (50 nM) in neuronal extracellular buffer with calcium or with Calcein $(1 \mu \mathrm{M})$ for $45 \mathrm{~min}$ in the dark after $30 \mathrm{~min}$ $\mathrm{CoCl}_{2}(1 \mathrm{mM})$ was added. Then cells were washed and imaged on a Leica DMI6000 Microscope. Imaging processing and data analysis were performed using LASAF software (Leica, Wetzlar, Germany). In some experiments cells were pre-incubated for $1 \mathrm{hr}$ with atractylate $(20 \mu \mathrm{M}$, Sigma), Cyclosporine A (CsA, $1 \mu \mathrm{M}$, Sigma), Bongkrekic acid (BkA, $10 \mu \mathrm{M}$, Sigma), FK-506 (5 $\mu \mathrm{M}$, Sigma), 0.1\% vehicle (DMSO), Tocopherol (50 $\mu \mathrm{M}, 4 \mathrm{hr}$, Sigma) or NAC (1 mM, 2 hr, Sigma).

For imaging expreriments, MEFs were cultured on glass bottom culture dishes. Cells were loaded for $\Delta \psi_{\mathrm{m}}$ with TMRM (50 nM) and Mitotracker Green (200 nM) (Molecular Probes, Life Technologies) with or without Oligomycin $(1 \mu \mathrm{M})$, FCCP $(1 \mu \mathrm{M})$ or $\mathrm{CoCl}_{2}(1 \mathrm{mM})$. For the mPTP opening assay, cells were loaded with calceinAM $(1 \mu \mathrm{M})$ and Mitotracker Red (150 nM) (Molecular Probes, Life Technologies), with or without $\mathrm{CoCl}_{2}$ (1 mM) both in HBSS $1 \mathrm{X}$ (Gibco, Life Technologies) for $20 \mathrm{~min}$ at $37^{\circ} \mathrm{C}$. Then, cells were washed three times in HBSS 1X. Live images of the cells were captured with the Olympus FluoView FV1000 Confocal Microscope (Olympus Imaging America Inc, Center Valley, PA, USA) and analyzed using ImageJ software.

\section{Oxidative stress assay}

To measure $\mathrm{H}_{2} \mathrm{O}_{2}$ production mitochondria were isolated using the mitochondrial isolation kit from Sigma according to the manufacturer instructions. The experiment was started by adding $100 \mu \mathrm{l}$ of assay buffer (HBSS containing $10 \mu \mathrm{M}$ Amplex Red, $10 \mathrm{mM}$ succinate, 0.2 units/ml Horse Radish Peroxidase) and followed over time on a fluorescence plate reader. The same conditions were used to determine the production of superoxide anion using the Dihydroethidium (10 $\mu \mathrm{M}$, DHEt) method. The protein carbonyl contents in cell lysates were detected by the OxyBlot protein oxidation detection kit (Millipore, Billerica, MA) using the instructions provided by the manufacturer. Lipid peroxidation was analyzed using the ThioBarbituric Acid Reactive Species (TBARS) assay and according to the manufacturer's instructions (Cayman Chemical, Ann Arbor, MI, USA).

\section{Analysis of mitochondrial morphology}

For visualization of mitochondria, MEFs were either stained with Mitotracker Red (250 nM) or infected with a retrovirus expressing mt-DsRed [25]. Primary cultured neurons were only imaged with MitoTracker Red (100 $\mathrm{nM})$. Then cells were washed for $10 \mathrm{~min}$ and fixed with 4\% PFA (Electron Microscopy Science, Hatfield, PA, USA) for $20 \mathrm{~min}$. After fixation coverslips were mounted on glass slides and imaged by epifluorescence on a Leica DMI6000 Microscope (Leica Microsystems GmbH, Wetzlar, Germany). For live imaging, after staining cells were mounted on a perfusion chamber in culture media containing HEPES $(1 \mathrm{mM})$ and imaged at $22^{\circ} \mathrm{C}$. Regardless of the staining method, cells were then scored by eye into four different categories according to the morphology of their mitochondrial network previously described [25]. The automatic analysis of the size and branching of the mitochondrial network was done using the particle analysis function of ImageJ according to a previously described methods using ImageJ [26].

\section{Calcium imaging}

FCCP releasable pool was measured by adapting a previously described method [27]. Briefly, MEFs and primary cortical neurons cultures were loaded with Fura-2 AM $\left(5 \mu \mathrm{M}, 45 \mathrm{~min}\right.$ at $37^{\circ} \mathrm{C}$ ) (Molecular probes, Life Technologies), and imaged with a Leica DMI6000 Microscope. Imaging processing and data analysis were performed using LAS AF software (Leica). FCCP (1 $\mu \mathrm{M})$ was applied using an 8-channel gravity perfusion system (ALA Scientific Instrument, Farmingdale, NY, USA).

\section{Statistical analysis}

Statistical analysis was performed using Prism 5 (GraphPad Software) and Excel (Microsoft). Pooled results were expressed as means \pm SEM. Significance was determined by the non paired Student $t$-test.

\section{Results}

Respiratory defects in intact mitochondria but normal activities of enzymes in the mitochondrial electron transport system (ETS) in PINK1-deficient cells

Our previous study revealed that respiratory activity is impaired in mitochondria isolated from striata or aged cortices of PINK1-/- mice [18]. To investigate further the underlying mechanisms, we derived mouse embryonic fibroblasts (MEFs) from PINK1-/- mice. MEFs 
allow the functional investigation of mitochondria in an intact cellular environment and are more amenable to experimental manipulations than mitochondria isolated from brains. We first examined whether intact mitochondria in PINK1-/- MEFs recapitulate the mitochondrial defects observed in isolated mitochondria from PINK1-/- striata. We measured the endogenous respiratory activity of primary MEFs energized with glucose (10 $\mathrm{mM})$. Consistent with our earlier in vivo findings, endogenous respiration rate is reduced in PINK1-/fibroblasts (Figure 1A and 1B).

Respiratory defects in PINK1-deficient cells have been suggested to result from impaired plasma membrane glucose transporter activity, which reduces substrates delivery to mitochondria [15]. To bypass the glucose transporter, we measured the respiratory activity of digitoninpermeabilized cells energized with $10 \mathrm{mM}$ glutamate/ malate (complex I substrate) or $10 \mathrm{mM}$ succinate (complex II substrate) or $1 \mathrm{mM}$ Ascorbate/1 mM TMPD (complex IV substrates) in the presence of saturating concentrations of ADP $(1 \mathrm{mM})$. The use of digitonin ( $0.01 \%$ final concentration) allows the direct delivery of substrates to mitochondria by specifically permeabilizing the plasma membrane without affecting mitochondrial integrity. Interestingly, we found that state 3 activities, which represent the maximum respiration rate in the presence of ADP are significantly decreased for complex I and complex II substrates in PINK1-/- fibroblasts (Figure $1 \mathrm{C}$ and 1D). These results are similar to what we and others have independently reported in PINK1-/mice and MEFs $[16,18]$, indicating that PINK1-/- MEFs represent a valid cellular model to study the detailed mechanisms underlying respiratory defects seen in PINK1-/- mice.

Oxidative phosphorylation is a complex process relying on the proper function of several enzymatic complexes and the maintenance of transmembrane potential $\left(\Delta \Psi_{\mathrm{m}}\right)$. We therefore measured the enzymatic activities of all individual complexes composing the electron transport system (ETS) in MEFs. Using spectrophotometric methods, we measured enzymatic activities for complex I (NADH-ubiquinone reductase activity), complex II (succinate-ubiquinone reductase activity), complex III (ubiquinol-Cytochrome $\mathrm{C}$ reductase activity) and complex IV activity (Cytochrome oxidase activity). After normalization to citrate synthase activity, enzymatic activities of all complexes composing the ETS appear normal in PINK1-/- MEFs (Figure 1E). Reduced respiration has been documented to result from coenzyme $Q$ deficiency, which affects the transfer of electrons from
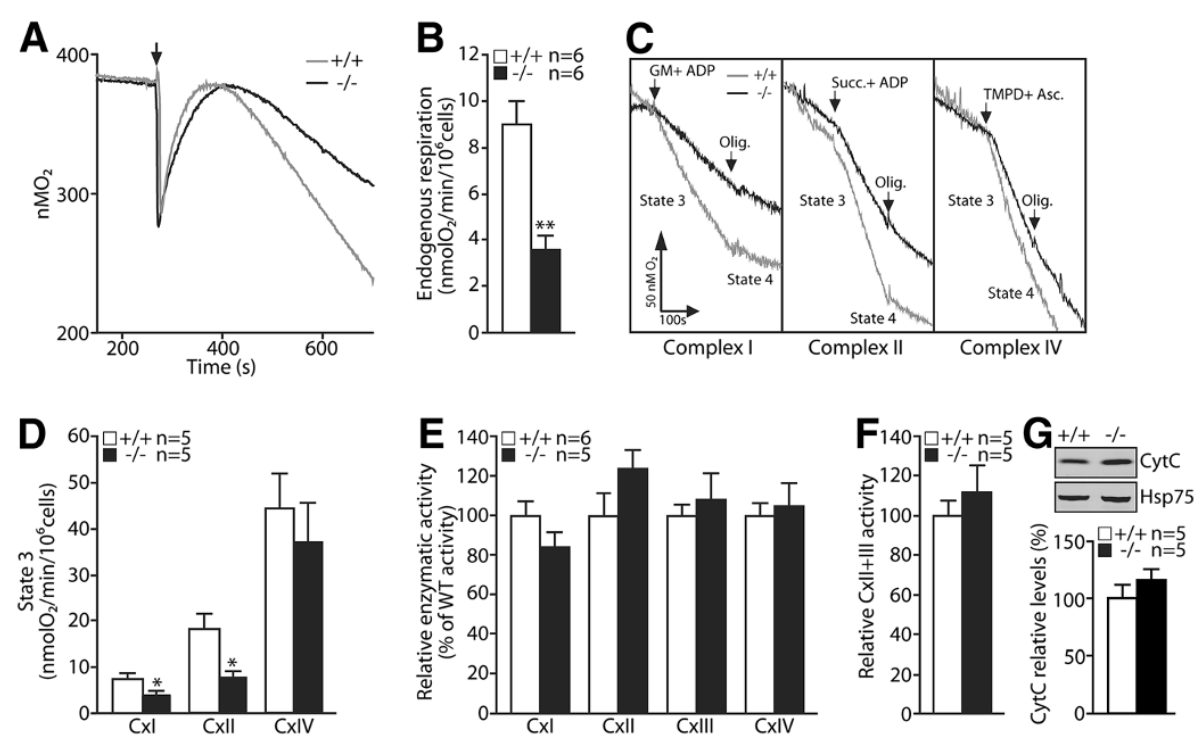

Figure 1 Impaired mitochondrial respiration in PINK1-/- fibroblasts. A. Representative oxygraphs of PINK1-/- (-/-) and wild-type (+/+) MEFs energized with glucose (10 mM). The arrow indicates the time MEFs are added to the chamber. B. Bars show oxygen consumption, which represents the endogenous respiratory activity in PINK1-/- and +/+ MEFs. C Representative oxygraphs of PINK1-/- and +/+ MEFs energized with 10 mM glutamate/malate (complex I substrate), 10 mM succinate (complex II substrate) or $1 \mathrm{mM}$ TMPD/1 mM ascorbate (complex IV substrate) in the presence of ADP $(1 \mathrm{mM})$. Arrows indicate the time of the addition of either the substrates or oligomycin (2 $\mu$ M). D. Bars show State 3 respiratory activity for complex I, II and IV in PINK1-/- and +/+ MEFs permeabilized with digitonin. E. Enzymatic activities of complexes I, II, III and IV of the mitochondrial electron transport system, as measured by spectrophotometric assays and after normalization to citrate synthase activity. F. Graph represents succinate-cytochrome C oxidase (complex II+ III) activity. G. Upper panel: Representative western blot showing levels of cytochrome $C$ in the mitochondrial fraction of PINK1-/- and +/+ MEFs. Hsp75 is used as a control for the total amount of mitochondrial proteins loaded in each well. Lower panel: The bar graph shows relative quantification of the level of Cytochrome C using Hsp75 as loading control. All data are expressed as mean \pm SEM. * $p<0.05,{ }^{* *} p<0.01$. 
complexes I or II to III [28]. We investigated a possible coenzyme $\mathrm{Q}$ deficiency by measuring the antimycin sensitive succinate-Cytochrome $\mathrm{C}$ reductase activity. This activity was also found normal in PINK1-/- MEFs (Figure 1F). Reduced Cytochrome $C$ in the intermembrane space results in reduced electron transfer between complex III and IV and impairs mitochondrial respiratory activities. We measured the level of Cytochrome $\mathrm{C}$ in the mitochondrial fraction of PINK1-/- MEFs and control cells and found that the levels were similar between the two genotypic groups after normalization using Hsp75 as loading control (Figure 1G).

\section{Reduced mitochondrial transmembrane potential of PINK1-/- MEFs}

In the absence of enzymatic defects of the ETS complexes, we turned our attention towards mitochondrial transmembrane potential $\left(\Delta \Psi_{\mathrm{m}}\right)$, the electrochemical force that modulates the kinetics of proton reentry to the matrix through ATP-synthase. Using microscopic and flow cytometric analyses, we measured the transmembrane potential of MEFs stained with TMRM (50 $\mathrm{nM}$ ). TMRM is a cationic fluorescent dye that accumulates inside the mitochondrial matrix according to the membrane potential. Interestingly, TMRM fluorescence signal is reduced in both experiments in PINK1-/MEFs (Figure 2A-D). To ensure that dye is equally loaded and that the TMRM signal is not auto-quenched we compared TMRM fluorescence in PINK1-/- and control MEFs following oligomycin and FCCP treatment. Oligomycin, an inhibitor of ATP synthase, induces hyperpolarization of mitochondria and increases of TMRM fluorescence, whereas FCCP dissipates transmembrane potential. No differences in TMRM fluorescence between the two genotypes were found following either of these treatments.

We investigated whether $\Delta \Psi_{\mathrm{m}}$ is similarly affected by the loss of PINK1 in primary cortical neurons. Because flow cytometry requires re-suspension of cells that could damage mature neurons, we measured $\Delta \Psi_{\mathrm{m}}$ using fluorescence microscopy. Neuronal cultures were stained with TMRM (50nM), imaged with a fluorescent microscope and the intensity of the TMRM fluorescence was determined for each individual neuron. Consistent with what we have observed in fibroblasts, TMRM fluorescence is reduced by $40 \%$ in PINK1-/- neurons, confirming a reduction of $\Delta \Psi_{\mathrm{m}}$ in the absence of PINK1 (Figure 2E).

\section{Increased mPTP opening in the absence of PINK1}

Since the enzymatic activity of each component of the ETS is normal, we looked for alternative mechanisms underlying the reduction of $\Delta \Psi_{\mathrm{m}}$. We evaluated opening of the mitochondrial permeability transition pore, which allows the diffusion of small ions across the mitochondrial inner membrane [29]. We compared opening of mPTP in PINK1-/- and control MEFs under basal conditions using the $\mathrm{CoCl}_{2}$-calcein fluorescence-quenching assay [30]. Calcein-AM is a membrane permeable fluorophore that diffuses freely into all subcellular compartments including mitochondria. The acetoxymethyl (AM) group of the fluorophore is cleaved by ubiquitous intracellular esterase. Calcein, which is hydrophilic, is then trapped within all subcellular compartments. The cells are then loaded with the divalent cobalt cation $\left(\mathrm{Co}^{2+}\right)$, which quenches calcein fluorescence in all subcellular compartments except the mitochondrial matrix, as the inner mitochondrial membrane is the only intracellular membrane that is normally $\mathrm{Co}^{2+}$-impermeable [30,31]. However, during the opening of the MPTP, cobalt enters mitochondria and is able to quench mitochondrial calcein fluorescence [32].

Under basal conditions, calcein fluorescence measured by both microscopic and flow cytometric analyses is lower in PINK1-/- MEFs, suggesting increases in $\mathrm{mPTP}$ opening (Figure 3A-D). We also measured calcein fluorescence in the absence of cobalt. As expected, calcein fluorescence is much higher in the absence of $\mathrm{Co}^{2+}$ and is similar between PINK1-/- and control cells, indicating similar calcein loading (Figure 3A-D). We extended the analysis to PINK1-/- neurons to confirm if $\mathrm{mPTP}$ opening is also increased. Because flow cytometric analysis requires re-suspension of cultured cells and thus would damage mature neurons, we used only microscopic analysis [33]. We found marked reduction of calcein fluorescence in PINK1-/- cortical neurons, further confirming increases in MPTP opening in the absence of PINK1 (Figure 3E).

We further tested whether treatment of atractylate, an agonist of the MPTP, would result in decreases of calcein fluorescence. Following the treatment, calcein signal is considerably reduced and the low level of calcein fluorescence is similar between the two genotypic groups in both MEFs and neurons (Figure 4A-D). These results provide additional support that the lower calcein signal in PINK1-/- cells is due to increased opening of the MPTP.

We further treated cells with two agents that can inhibit opening of the MPTP. Treatment with cyclosporin A (CsA), an inhibitor of MPTP, eliminated the genotypic difference in calcein fluorescence between PINK1-/and control MEFs and neurons (Figure 4). Although CsA is a strong inhibitor of the MPTP [34], it has additional molecular targets such as calcineurin [35]. We therefore used another mPTP inhibitor, bongkrekic acid, which inhibits the mitochondrial ATP-ADP translocase, a component of the mPTP, and has no inhibitory effect on calcineurin [36]. Treatment with bongkrekic acid also abrogates the genotypic difference in MEFs and neurons 

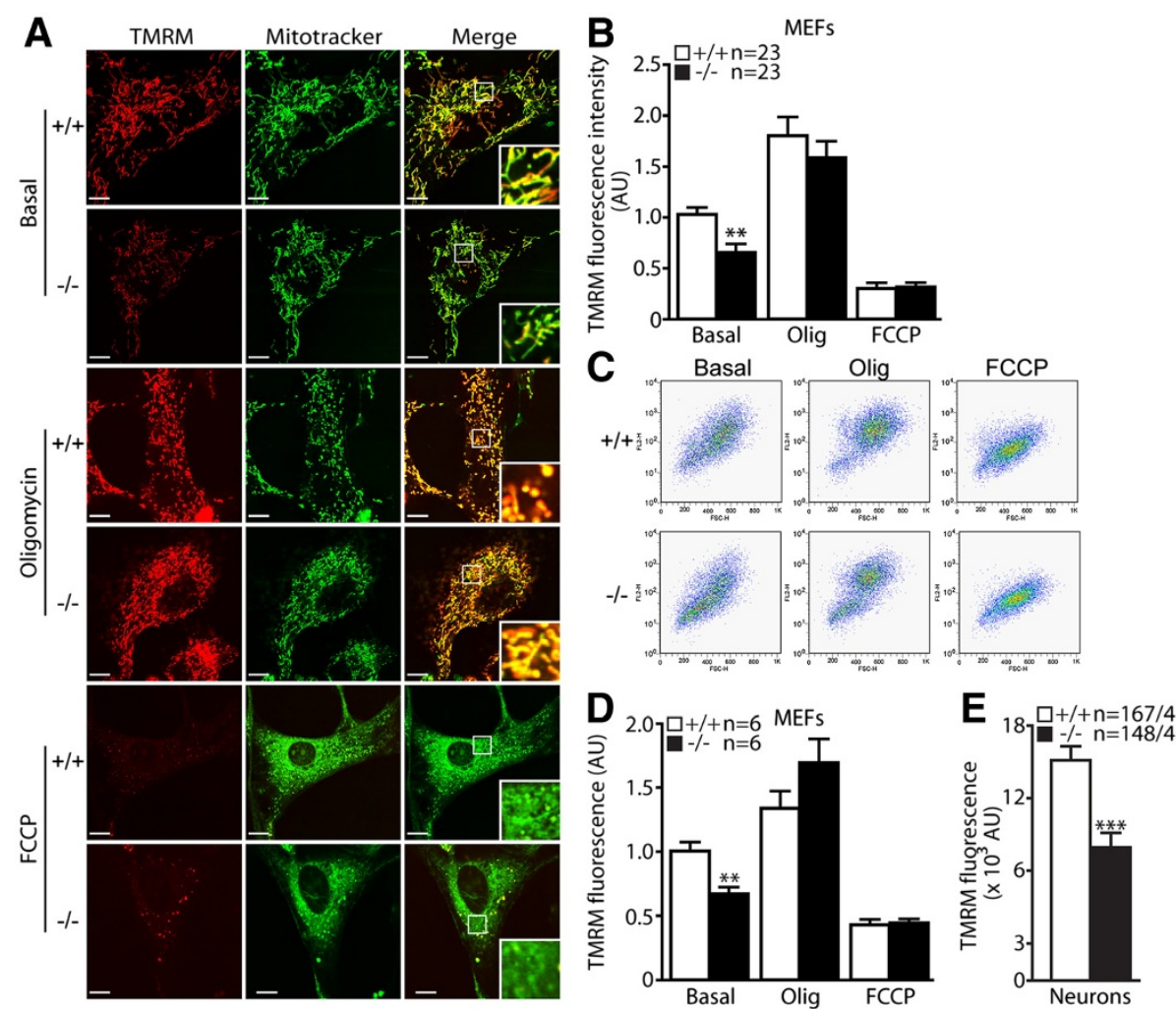

Figure 2 Reduced mitochondrial transmembrane potential in PINK1-/- cells. A. Representative confocal microscopic images of PINK1-/and $+/+$ MEFs after staining with TMRM (50 nM, red) and Mitotracker Green (200 nM) in the presence or absence of oligomycin (Olig, $1 \mu M)$ or FCCP $(10 \mu \mathrm{M})$. The intensity of TMRM reflects the level of mitochondrial transmembrane potential, whereas the intensity of Mitotracker Green is not affected by transmembrane potential. The bottom right inserts are the higher power views of the boxed areas in the same panel. The TMRM signal is reduced in PINK1-/- cells, whereas the TMRM signal is increased and decreased similarly in both PINK1-/- and +/+ cells following oligomycin and FCCP treatment, respectively. B. The bar graph shows quantification of TMRM signals in PINK1-/- and +/+ MEFs in the presence or absence of oligomycin and FCCP using confocal images. The number shown in the panel indicates the number of cells used in the study. $\mathbf{C}$. Representative flow cytometry dot plots show the intensity of TMRM signals in PINK1-/- and +/+ MEFs following incubation with TMRM (50 nM) in the presence or absence of oligomycin $(1 \mu \mathrm{M})$ or FCCP $(10 \mu \mathrm{M})$. D. The bar graph shows quantification of TMRM signals measured by FACS analysis in PINK1-/- and +/+ MEFs. The number shown in the panel indicates the number of independent experiments performed. $\mathbf{E}$. The bar graph of TMRM fluorescent signals in PINK1-/- and +/+ neurons shows reduced TMRM signals in PINK1-/- neurons. The numbers shown indicate the number of neurons used (left) and the number of independent experiments performed (right) in the study. All data are expressed as mean \pm SEM. Scale bar: $10 \mu \mathrm{m} .{ }^{*} p<0.05,{ }^{* *} p<0.01,{ }^{* * *} p<0.001$

(Figure 4). Furthermore, treatment of cells with FK-506, a potent calcineurin inhibitor that has no direct effect on the MPTP [37], showed that it has little effect on calcein signals (Figure 4). Using fluorescence based enzymatic assay we confirmed that treatment with FK-506 $(5 \mu \mathrm{M})$ results in an almost complete inhibition of calcineurin activity (data not shown). These results provide further support that loss of PINK1 results in increased opening of the MPTP.

Inhibition of the MPTP opening rescues the reduction of mitochondrial transmembrane potential and impairment of mitochondrial respiration in PINK1-/- cells

To investigate whether increased opening of $\mathrm{mPTP}$ underlies the mitochondrial functional defects in PINK1 $-/$ - cells, we treated cells with MPTP agonist, atractylate, as a positive control of the effects of $\mathrm{mPTP}$ opening on mitochondrial depolarization. Treatment with atractyloside acid results in a $64 \%$ reduction of TMRM fluorescence in wild-type control cells compared to basal conditions, indicating a strong depolarization of mitochondrial inner membrane (Figure 5A and 5B).

We then treated the cells with MPTP inhibitors to determine whether inhibition of $\mathrm{MPTP}$ opening can rescue the transmembrane potential deficits in PINK1-/- cells. Indeed CsA treatment fully rescued the reduced TMRM fluorescence in PINK1-/- MEFs (Figure 5). Bongkrekic acid also partially rescued the reduced TMRM fluorescence in PINK1-/- MEFs (Figure 5). FK-506 treatment had no effect on TMRM fluorescence (Figure 5). Furthermore, cobalt treatment did not alter TMRM signals in PINK1-/- and +/+ MEFs, indicating that cobalt does 

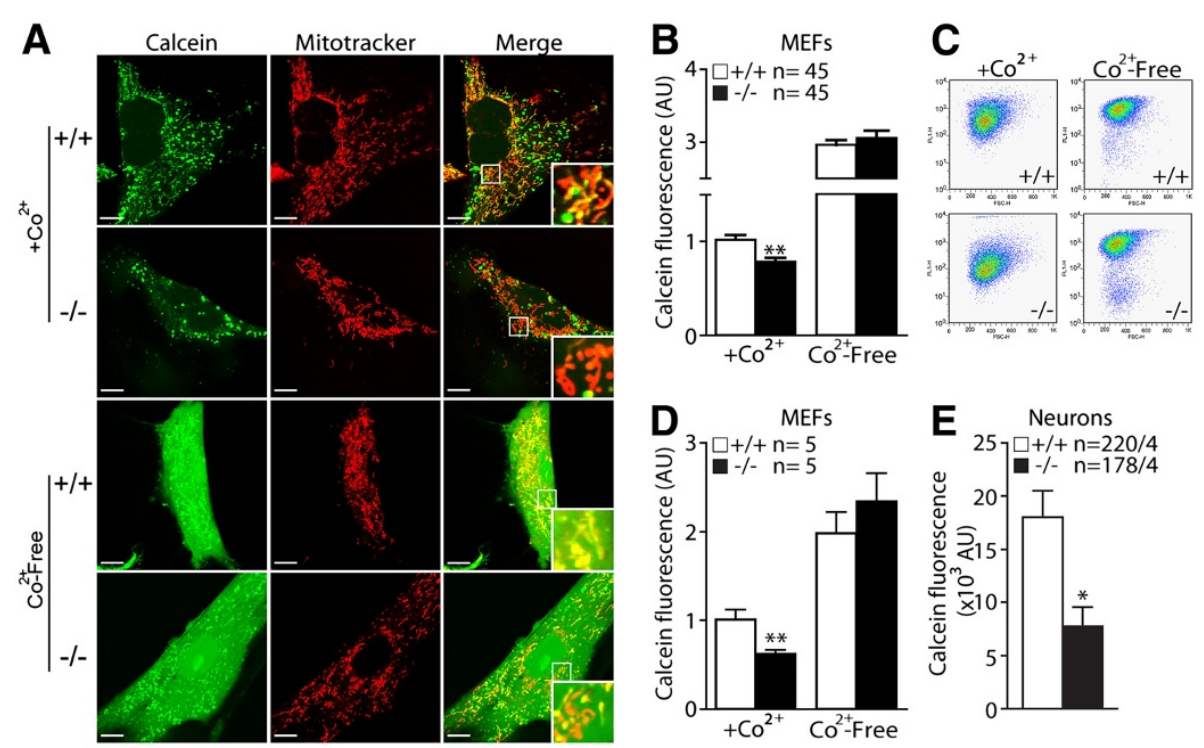

Figure 3 Increased opening of the mitochondrial permeability transition pore in PINK1-/- cells. A. Representative confocal microscopic images of PINK1-/- and +/+ MEFs after incubation with calcein-AM (1 $\mu \mathrm{M})$ and Mitotracker Deep Red (150 nM) in the presence or absence of $\mathrm{Co}^{2+}(1 \mathrm{mM})$, which quenches calcein fluorescence (green) outside of mitochondria. Mitotracker Deep Red allows visualization of calcein fluorescence in mitochondria. The bottom right inserts are the higher power views of the boxed areas in the same panel. The calcein fluorescence in mitochondria is lower in PINK1-/- cells in the presence of $\mathrm{CO}^{2+}$. In the absence of $\mathrm{Co}^{2+}$, calcein fluorescent signals are very intense and are present in the entire cell, and there are no genotypic differences. B. The bar graph shows quantification of calcein fluorescence in PINK1-/- and +/+ cells in the presence or absence of $\mathrm{CO}^{2+}$ using confocal images. The number shown in the panel indicates the number of cells used in the study. C. Representative flow cytometry dot plots show the intensity of calcein signals in PINK1-/- and +/+ MEFs following incubation with calcein-AM $(1 \mu \mathrm{M})$ in the presence or absence of $\mathrm{Co}^{2+}(1 \mathrm{mM})$. D. The bar graph of calcein signals measured by FACS analysis shows reduced calcein signals in PINK1-/- MEFs in the presence of $\mathrm{CO}^{2+}$. The number shown in the panel indicates the number of independent experiments performed. E. The bar graph of calcein fluorescent signals in PINK1-/- and +/+ neurons shows reduced calcein signals in PINK1-/neurons. The numbers shown indicate the number of neurons used (left) and the number of independent experiments performed (right) in the study. All data are expressed as mean \pm SEM. Scale bar: $10 \mu \mathrm{m} .{ }^{*} p<0.05,{ }^{* *} p<0.01$.

not affect mitochondrial membrane potential in our experimental time frames (Figure 5). These results show that inhibition of $\mathrm{mPTP}$ opening rescues the reduction in mitochondrial transmembrane potential in the absence of PINK1, suggesting that increased opening of the mPTP underlies reduction of $\Delta \Psi \mathrm{m}$.

We further tested whether inhibition of mPTP opening reverses the mitochondrial respiration impairment in PINK1-/- cells. Treatment with CsA reduced the genotypic difference between PINK1-/- and control cells to the extent that endogenous respiratory activity is similar (Figure 6A and 6B). Moreover, CsA treatment almost fully rescued complex I driven respiration in PINK1-/cells (Figure 6C and 6D). Treatment with FK-506 was not able to rescue the respiratory impairment in PINK1 $-/-$ cells, indicating that the effect of CsA on respiration was specific for its inhibitory effect on MPTP (Figure 6E and $6 \mathrm{~F})$.

\section{Normal levels of oxidative stress in PINK1-/- cells}

Because MPTP opening can be affected by elevated oxidative stress [29], we went further to examine whether there is an accumulation of oxidative species in the mitochondrial fraction of PINK1-/- and control MEFs. We measured the levels of protein carbonyls, a marker of protein oxidation. As measured by OxyBlot, the total level of carbonyls is similar between the two genotypic groups (Figure 7A). We then measured the accumulation of another common marker of oxidative stress, thiobarbituric acid reactive substances (TBARS), which reflects lipid peroxidation, and found no significant differences between the two genotypes (Figure 7B). We further evaluated the production of oxidative species. Using the Amplex Red dye fluorescence assay we evaluated the propensity of cells to generate Reactive Oxygen Species (ROS) by measuring the production of $\mathrm{H}_{2} \mathrm{O}_{2}$. Because $\mathrm{H}_{2} \mathrm{O}_{2}$ extrusion across the plasma membrane can be kinetically limiting we measured the rate of $\mathrm{H}_{2} \mathrm{O}_{2}$ produced by isolated mitochondria from MEFs. Mitochondria are the main source of ROS in the cells. We found that isolated mitochondria from PINK1-/and WT cells energized with succinate $(10 \mathrm{mM})$ produce $\mathrm{H}_{2} \mathrm{O}_{2}$ at similar rates (Figure $7 \mathrm{C}$ ). We also monitored the production of superoxide anion $\mathrm{O}_{2}^{-}$ Superoxide is the primary oxidant species generated as a byproduct of mitochondrial respiration. Using the 

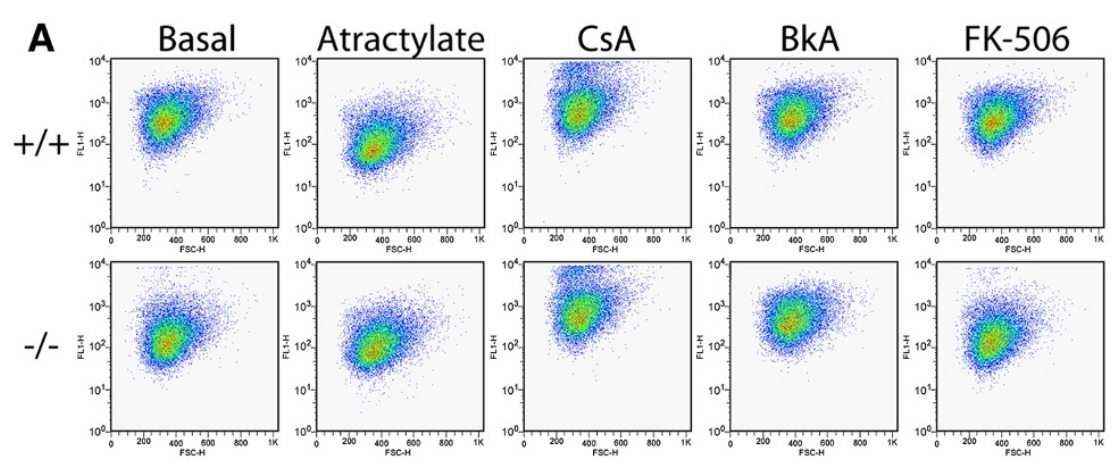

B
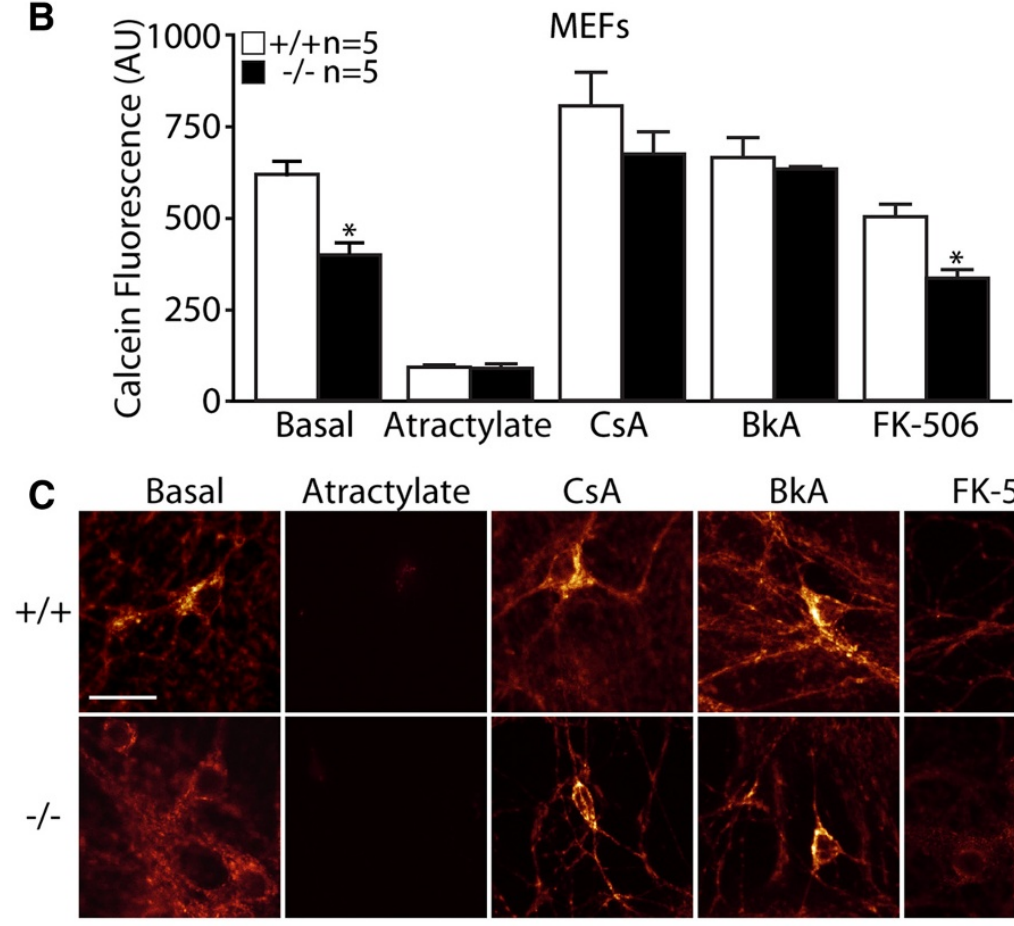

Atractylate

CsA
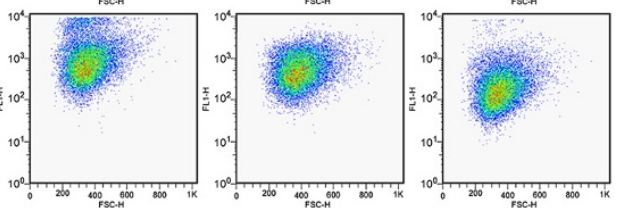

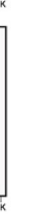


DHEt dye fluorescence assay, we found similar kinetics of $\mathrm{O}_{2}^{--}$generation between PINK1-/- and WT MEFs (Figure 7D). As positive controls we used MEFs derived from our $D J-1-/-$ mice. Using the same assay conditions, DJ-1 MEFs displayed higher rates of $\mathrm{H}_{2} \mathrm{O}_{2}$ and $\mathrm{O}_{2}^{-}$production as monitored with the Amplex Red and the DHEt assays (data not shown). Thus, loss of PINK1 does not increase the production of reactive oxygen species.

We further evaluated the effect of antioxidant treatments on mPTP opening. Cells were treated with Tocopherol and N-acetyl-Cysteine. Tocopherol is a cell permeable antioxidant, which reduces lipid oxidative stress and has been shown to prevent mPTP opening in conditions of elevated oxidative stress [38]. N-AcetylCysteine is a cell permeable precursor of glutathione, which can prevent oxidative stress induced mPTP [39]. Calcein fluorescence levels remained reduced in PINK1 -/ - MEFs compared to control cells following antioxidant treatment (Figure 7E and 7F). These results suggest that increased mPTP opening in PINK1-/- MEFs unlikely results from elevated oxidative stress.

\section{Normal mitochondrial morphology in PINK1-deficient cells} Given the earlier reports on the effects of PINK1 inactivation in mitochondrial fusion $[9,40,41]$ and fission $[10,13,42]$ in fruit flies and mammalian cell lines, we examined whether mitochondrial morphology is affected in PINK1-/- MEFs and neurons, which could contribute to the functional deficits we observed in these cells. We assessed mitochondrial morphology in PINK1-/- and wild-type MEFs using a previously established method [25]. MEFs were transfected with the mitochondria targeted fluorescent protein mt-dsRed then analyzed by distributing cells into four different categories according to the morphology of their mitochondrial network: (I) cells with a tubular mitochondrial network and less than 5 small round mitochondria; (II) cells with more than $50 \%$ tubular mitochondria; (III) cells with more than $50 \%$ small and spherical mitochondria; (IV) cells with a fragmented mitochondrial network and less than 5 tubular mitochondria. Under basal conditions, PINK1-/and WT MEFs show similar distribution among the four categories (Figure 8A). To circumvent any possible artifacts due to overexpression of a mitochondrial protein, we performed the same analysis using MitoTracker Red on fixed cells and still did not observe any significant differences between the two genotypic groups (Figure $8 \mathrm{~B}$ ). A similar study on primary cortical neurons derived from PINK1-/- and WT mice also showed comparable tubularity of the mitochondrial network in both genotypic groups (Figure 8C).

We next sought to assess mitochondrial morphology in a more quantitative manner. We quantified the number and the average size of mitochondria in each cell using ImageJ on binary images (Figure 8D panels $\mathrm{i}$ and ii) of MitoTracker Red stained cells as previously described [26]. We did not observe significant differences between genotypes in these two parameters (Figure 8E). We further quantified the number of branches and junctions per mitochondrion using mitochondrial skeleton images, and did not observe any significant differences between the
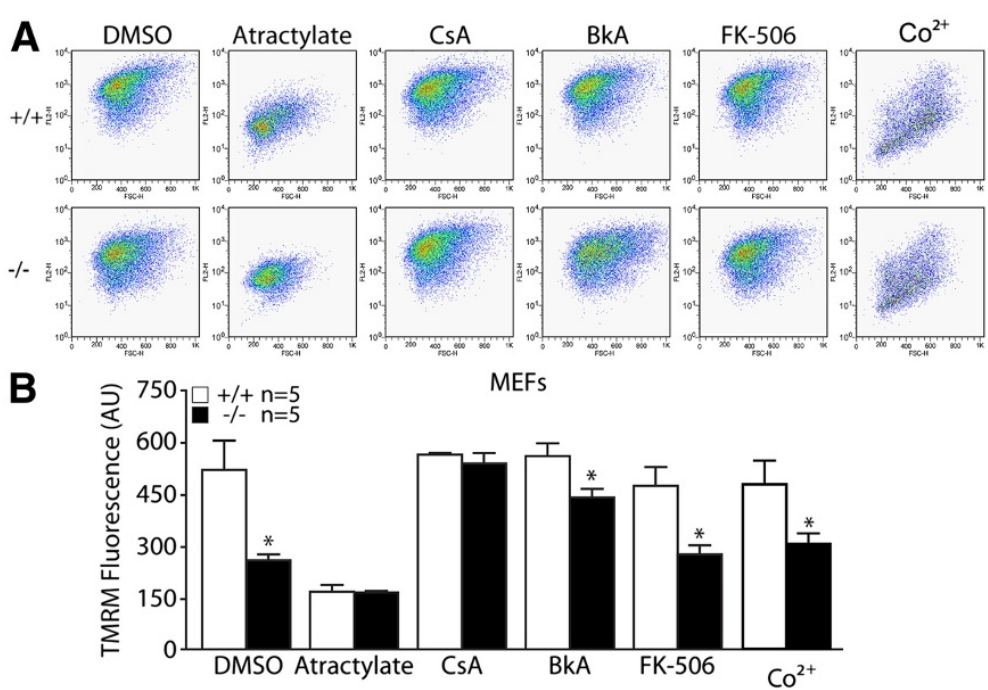

Figure 5 Blockade of mPTP opening attenuates the defect in mitochondrial transmembrane potential in PINK1-/- MEFs. A. Representative flow cytometry dot plots showing TMRM fluorescence in PINK1-/- and +/+ cells incubated with TMRM (50 nM) and atractylate $(20 \mu \mathrm{M}), \mathrm{Cs} A(1 \mu \mathrm{M}), \mathrm{BkA}(10 \mu \mathrm{M})$, FK-506 $(5 \mu \mathrm{M})$ or $\mathrm{Co}^{2+}(1 \mathrm{mM})$. B. The bar graph shows mitochondrial membrane potential measured by TMRM fluorescence after FACS analysis following various treatment, atractylate $(20 \mu \mathrm{M}), \operatorname{CsA}(1 \mu \mathrm{M}), \mathrm{BKA}(10 \mu \mathrm{M}), \mathrm{FK}-506(5 \mu \mathrm{M})$ or Co ${ }^{2+}(1 \mathrm{mM})$. The number shown in the panel indicates the number of independent experiments performed. All data are expressed as mean \pm SEM. ${ }^{*} p<0.05$. 

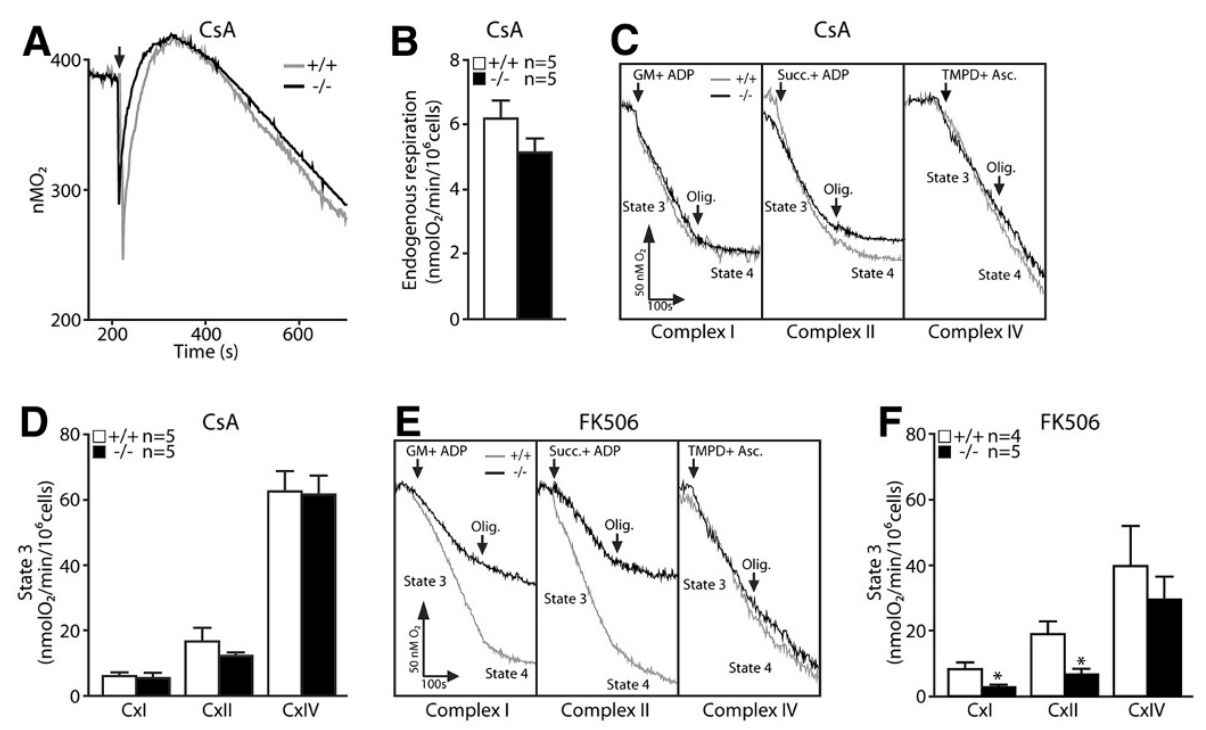

Figure 6 Blockade of mPTP opening by CsA attenuates the respiratory defect in PINK1-/- MEFs. A. Representative oxygraphs of PINK1-/and $+/+$ MEFs energized with glucose $(10 \mathrm{mM})$ in the presence of CSA $(1 \mu \mathrm{M})$. The arrows indicate the time MEFs are added to the chamber. $\mathbf{B}$. Oxygen consumption, which represents the endogenous respiratory activity in PINK1-/- and +/+ MEFs after treatment with CSA (1 $\mu$ M). C. Representative oxygraphs of PINK1-/- and +/+ MEFs energized with $10 \mathrm{mM}$ glutamate/malate (complex I substrate), $10 \mathrm{mM}$ succinate (complex II substrate) or $1 \mathrm{mM}$ TMPD/1 mM ascorbate (complex IV substrate) in the presence of CsA (1 $\mu \mathrm{M})$. Arrows indicate the time of the addition of either the substrate or oligomycin $(2 \mu \mathrm{M})$. D. Graph showing State 3 respiratory activity for complex I, complex II and complex IV in PINK1-/- and +/+ MEFs permeabilized with digitonin after treatment with CsA (1 $\mu \mathrm{M})$. E. Representative oxygraphs of PINK1-/- and +/+ MEFs energized with $10 \mathrm{mM}$ glutamate/malate (complex I substrate), $10 \mathrm{mM}$ succinate (complex II substrate) or $1 \mathrm{mM}$ TMPD/1 mM ascorbate (complex IV substrate) after treatment with FK-506 (5 $\mu \mathrm{M})$. Arrows indicate the time of the addition of either the substrate or oligomycin $(2 \mu \mathrm{M})$. F. Graph showing State 3 respiratory activity for complex I, complex II and complex IV in PINK1-/- and +/+ MEFs permeabilized with digitonin after treatment with FK$506(5 \mu \mathrm{M})$. All data are expressed as mean \pm SEM. ${ }^{*} p<0.05$.

genotypes (Figure 8E). Thus, loss of PINK1 does not affect mitochondrial morphology in primary cultured MEFs and neurons, at least under our culture conditions. These results suggest that the functional deficits observed in PINK1-/- MEFs and neurons are unlikely due to altered mitochondrial morphology.

\section{Increased mitochondrial calcium concentrations in PINK1- deficient cells}

We also evaluated mitochondrial calcium in the absence of PINK1, since elevated mitochondrial calcium levels are known to induce the opening of the MPTP [43]. We measured the size of the mitochondrial calcium pool by quantifying the amount of calcium released from mitochondria to the cytosol following FCCP treatment $(1 \mu \mathrm{M})$. FCCP is a specific proton ionophore that dissipates proton gradients and allows cations to freely redistribute across membranes according to their concentration gradients. Following FCCP treatment, alterations of cytosolic calcium concentrations were monitored with Fura-2, a BAPTA based dye whose fluorescence excitation ratio at $340 \mathrm{~nm}$ and $387 \mathrm{~nm}$ are proportional to cytosolic calcium concentrations. Increases in Fura-2 signals following FCCP treatment are much higher in PINK1-/- MEFs (Figure 9A and 9B) and neurons (Figure 9C and 9D) compared to their respective wild-type controls. These results suggest loss of PINK1 results in selective increase in mitochondrial calcium.

\section{Discussion}

In the current study, we investigated the mechanism underlying the mitochondrial respiration defects caused by loss of PINK1. We established primary MEFs and cortical neuronal cultures from our PINK1-/- mice to evaluate mitochondrial functions in intact cells. Similar to what we previously reported in mitochondria isolated from mouse brains [18], mitochondrial respiration is impaired in PINK1-/- MEFs, indicating that these cells represent a valid cellular model to study the detailed mechanisms underlying respiratory defects seen in PINK1-/- mice (Figure 1). Although respiration impairment can be caused by defects in mitochondrial transmembrane potential or the electron transport system, we found that only the mitochondrial transmembrane potential is reduced in PINK1-/- cells (Figure 2), while enzymatic activities of the complexes composing the electron transfer system are unaffected (Figure 1). In search for mechanisms underlying the reduction of the transmembrane potential, we found that opening of the mitochondrial permeability transition pore is increased 

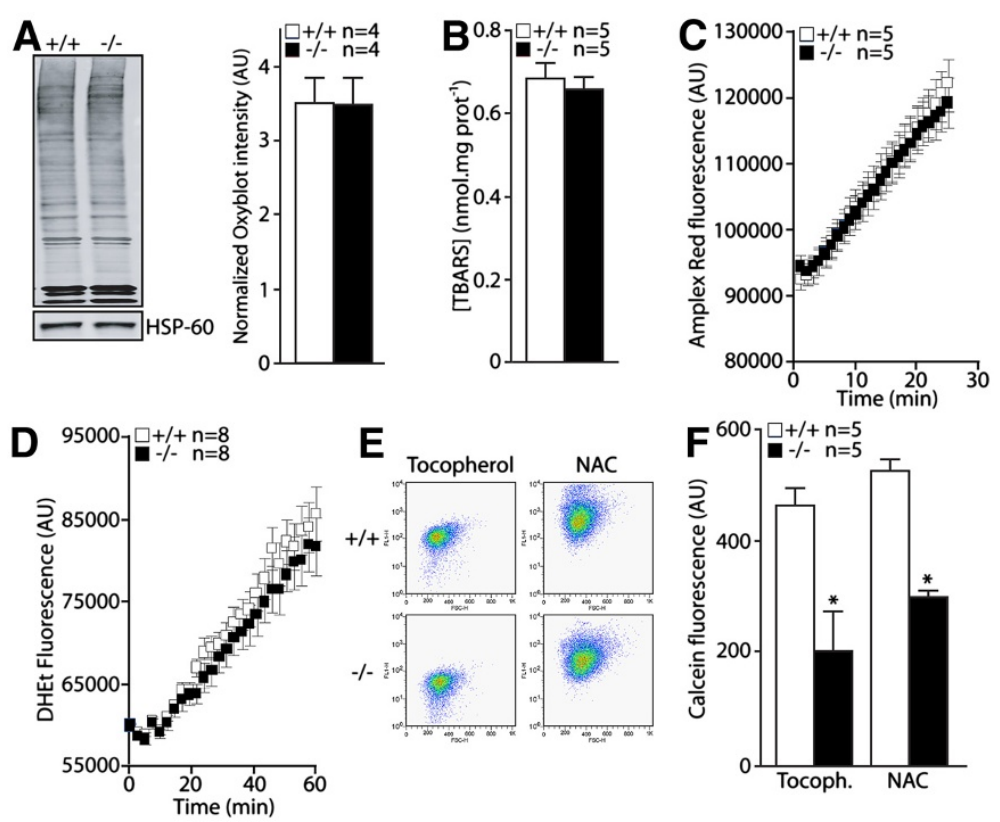

Figure 7 Normal levels of oxidative stress markers and normal production of reactive oxygen species in PINK1-/- MEFs. A. Left panel: OxyBlot analysis of mitochondrial fractions showing levels of protein carbonyls in both genotypes. Right panel: Quantification of levels of protein carbonyls in PINK1-/- and +/+ MEFs. B. Levels of lipid peroxidation measured by the TBARS assay in PINK1-/- and $+/+$ MEFs. C. Kinetics of $\mathrm{H}_{2} \mathrm{O}_{2}$ production in isolated mitochondria measured by following Amplex Red fluorescence over time. D. Kinetics of cytosolic superoxide anion $\mathrm{O}_{2}^{-}$ production measured in resuspended MEFs by following DHEt fluorescence over time. E. Representative flow cytometry dot plots showing calcein fluorescence in PINK1-/- and +/+ MEFs in the presence of $\mathrm{Co}^{2+}$ after antioxidant treatment with Tocopherol (50 $\left.\mu \mathrm{M}, 4 \mathrm{hr}\right)$ or NAC (1 mM, 2 hr). F. Quantification of calcein fluorescence in PINK1-/- and +/+ MEFs following treatment with Tocopherol (50 $\mu$ M, 4 hr) or NAC (1 mM, 2 hr). All data are expressed as mean \pm SEM. ${ }^{*} p<0.05$.

in the absence of PINK1 and that this defect can be rescued by inhibitors of the MPTP (Figures 3 and 4). Furthermore, mitochondrial transmembrane potential and respiration defects caused by loss of PINK1 were also reversed specifically by inhibitors of the MPTP (Figures 5 and 6), suggesting that increased opening of the MPTP underlies the defects in mitochondrial transmembrane potential and respiration observed in PINK1-/- cells. These mitochondrial functional defects occur in the absence of elevated oxidative stress (Figure 7) and mitochondrial morphological changes (Figure 8), but mitochondrial calcium is increased in PINK1-/- cells, suggesting that elevated mitochondrial calcium underlies the increase in MPTP opening (Figure 9).

Following our initial report of mitochondrial respiration defects in PINK1-/- mouse brains [18], a growing consensus has been building on the importance of PINK1 in mitochondrial respiration [15-19,44], though the underlying mechanism remained unclear. Defects in the activity of the electron transport system complexes have been suggested as a possible mechanism underlying the respiratory defects resulting from the loss of PINK1, as silencing PINK1 expression by siRNA in SH-SY5Y cells affected mitochondrial ATP synthesis and activity of ETC complexes [19]. However, enzymatic activities of the ETC complexes in our primary PINK1-deficient cells are normal. Instead we found that loss of PINK1 increased opening of the mitochondrial permeability transition pore, and that blocking MPTP opening occluded the difference between PINK1-/- and control MEFs for endogenous and State 3 respiration. These results suggest that increased $\mathrm{MPTP}$ opening is responsible for reduced mitochondrial respiration in PINK1-/cells. Previous reports showed that $\mathrm{mPTP}$ opening triggered by elevated calcium concentrations leads to reduced state 3 respiratory activities [45,46], an effect that can be prevented by pretreatment with CsA $[45,47,48]$.

Reduced transmembrane potential in PINK1-deficient cells has been reported in a wide variety of cells [15$17,21,49]$. In accordance with these previous reports, we also found that $\Delta \Psi \mathrm{m}$ is reduced in primary PINK1-/fibroblasts and neurons. It has been proposed that reduced enzymatic capacity of complex I of the mitochondrial electron transport system might be the underlying cause of the defects in $\Delta \Psi \mathrm{m}$ [11]. However, similar to other previous studies $[17,50,51]$, we found that complex I enzymatic activity as well as the activity of all other complexes composing the ETS are normal in our cell models in the absence of PINK1. Rather, we found 
A
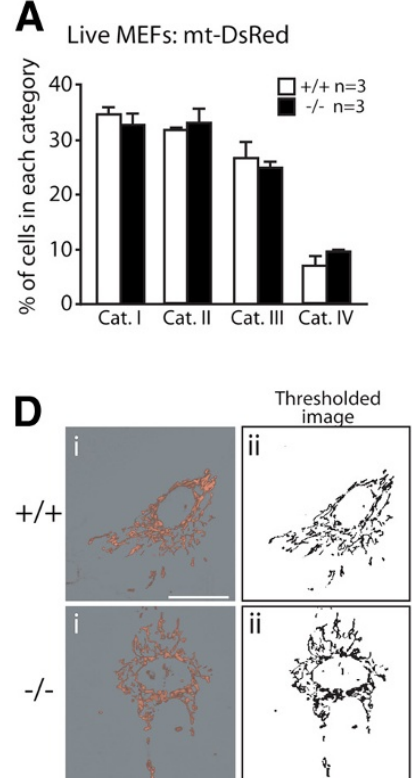

B $_{\text {Fixed MEFs: MitoTracker Red }}$

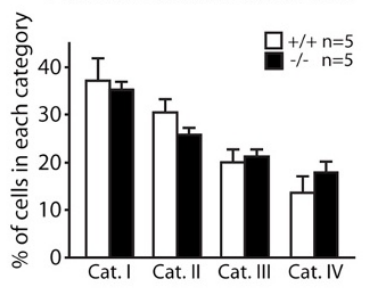

E

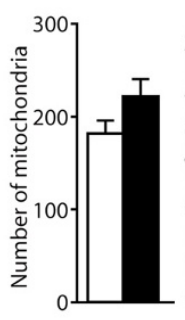

C $_{\text {Fixed Neurons: MitoTracker red }}$

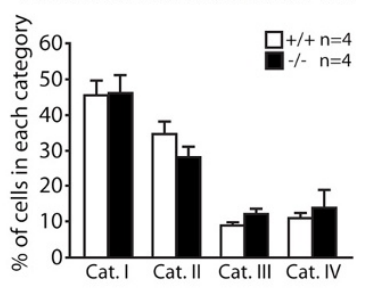

Cat.I Cat. II Cat. III Cat. IV

Figure 8 Unaltered mitochondrial morphology in PINK1-/- MEFs and neurons. A. Distribution of live PINK1-/- and $+/+$ MEFs infected with Mt-DsRed to the four morphological categories I, II, III and IV. B. Distribution of fixed PINK1-/- and +/+ MEFs stained with MitoTracker Red (250 $\mathrm{nM})$ to the four morphological categories. C. Distribution of fixed PINK1-/- and +/+ cortical neurons stained with MitoTracker Red (100 nM) to the four morphological categories. D. Representative fluorescent images of mitochondria stained with MitoTracker Red (250 nM) i) and the resulting binary (ii) used for quantitative analysis. E. Quantitative analysis using ImageJ shows the size and the number of branches of mitochondria in PINK1-/- and +/+ MEFs ( $P>0.05$ for all parameters measured). All data are expressed as mean \pm SEM. The numbers shown indicate the number of independent experiments performed in the study. For each experiment 100 cells were analyzed quantitatively.

that increased opening of the mPTP likely plays an important role in the mitochondrial depolarization observed in PINK1-/- cells, as inhibitors of the MPTP, CsA and BkA, rescued the $\Delta \Psi \mathrm{m}$ defects in PINK1-/cells. The opening of the MPTP allows free diffusion of small ions across the mitochondrial inner membrane as a corrective mechanism for cation overload [52-54]. Hence, increased opening of the MPTP allows a partial depolarization of the mitochondrial membrane, and this defect can be reversed by inhibition of $\mathrm{MPTP}$ opening such as CsA [24,52]. The stronger rescuing effect observed with CsA might relate to the fact that, in addition to blocking the MPTP, it also hampers mitochondrial calcium uptake, which may be increased in PINK1-deficient cells, as suggested by higher mitochondrial calcium levels in these cells.

A possible role of PINK1 in modulating mitochondrial morphology and dynamics emerged from studies in Drosophila. Loss of PINK1 function in flies results in abnormally large mitochondria with fragmented cristae and reduced capacity to generate ATP [6,7]. This mitochondrial phenotype is suppressed by genetically promoting mitochondrial fission or decreasing mitochondrial fusion, inferring that perturbed mitochondrial fission in PINK1deficient models underlies functional defects $[9,40,41]$. However, whether and how PINK1 may regulate mitochondrial morphology and dynamics in mammalian cells is much less clear. The effects of PINK1 deficiency on mitochondrial morphology and dynamics seem to depend on the cell type studied and range from inducing mitochondrial fission [10,42] or fusion [14] to no effect $[11,16,19,50]$. Consistent with these studies $[11,16,19,50]$, our analysis of primary cultured PINK1-/- MEFs and neurons did not show overt changes in mitochondrial morphology in fixed or live cells (Figure 8). These findings are also in agreement with our earlier EM study showing that no drastic ultrastructural changes in mitochondrial number and integrity in PINK1-/- brains at 3-4 and 2224 months of age [18]. Thus, loss of PINK1 function causes mitochondrial functional defects, in the absence of morphological changes, suggesting that the morphological abnormalities observed in mammalian cell lines may be downstream consequences of these mitochondrial functional defects.

How does loss of PINK1 lead to increased mPTP opening? Opening of the MPTP is primarily induced by oxidative stress and/or elevated intramitochondrial calcium concentrations [45]. We did not find any evidence of oxidative damage or increased production of ROS in PINK1-deficient cells (Figure 7). However, we found that mitochondrial calcium concentration measured indirectly in the cytosol following FCCP treatment is increased in PINK1-/- MEFs and neurons (Figure 9). This observation is consistent with a recent study 

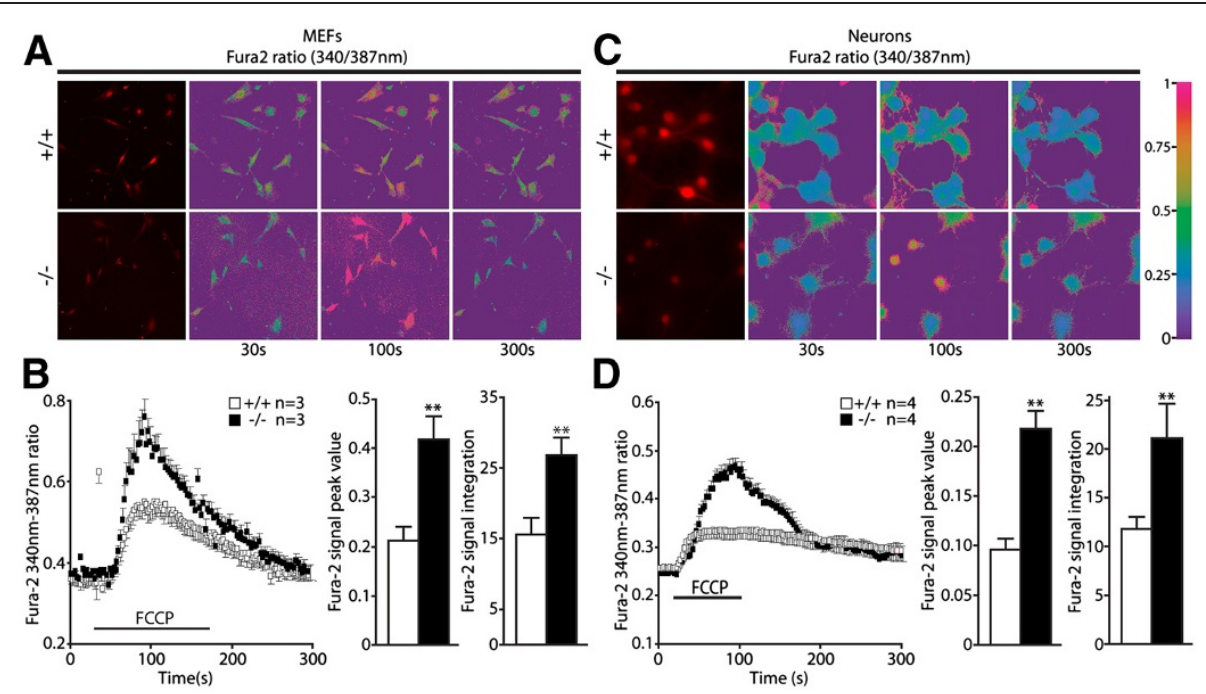

Figure 9 Higher mitochondrial calcium levels in PINK1-/- cells. A. Representative Fura-2 images of $\mathrm{Ca}^{2+}$ responses following FCCP treatment in PINK1-/- and +/+ MEFs. Fura-2 ratios at 340/387 are shown at time points indicated. The pseudocolor calibration scale for 340/387 ratios is shown on the right. FCCP $(1 \mu \mathrm{M})$ was added at $\mathrm{t}=25 \mathrm{~s}$. B. Left panel: Time course of cytosolic $\left[\mathrm{Ca}^{2+}\right]$ rise following FCCP treatment in PINK1-/and +/+ MEFs. Middle panel: The peak value of cytosolic calcium rise following FCCP is higher in PINK1-/- cells. Right panel: The average size of the FCCP releasable $\mathrm{Ca}^{2+}$ pool is higher in PINK1-/- MEFs compared to controls. C. Representative Fura-2 images of $\mathrm{Ca}^{2+}$ responses following FCCP treatment in PINK1-/- and +/+ neurons. D. Left panel: Time course of cytosolic $\left[\mathrm{Ca}^{2+}\right]$ rise following FCCP treatment in PINK1-/- and +/+ neurons. Middle panel: The peak value of cytosolic calcium rise following FCCP is higher in PINK1-/- neurons. Right panel: The average size of the FCCP releasable $\mathrm{Ca}^{2+}$ pool is higher in PINK1-/- neurons. All data are expressed as mean \pm SEM. ${ }^{* *} p<0.01$.

showing that loss of PINK1 reduces the activity of the mitochondrial $\mathrm{Na}^{+} / \mathrm{Ca}^{2+}$ exchanger (NCX), which regulates $\mathrm{Na}^{+}$-dependent $\mathrm{Ca}^{2+}$ efflux [15]. Pharmacologic inhibition of NCX activity leads to accumulation of calcium in isolated mitochondria [55]. It is therefore possible that impaired NCX activity in PINK1-/- cells may lead to accumulation of mitochondrial calcium, which in turn increases the opening of the MPTP. In this context, the MPTP may serve as a $\mathrm{Ca}^{2+}$-activated $\mathrm{Ca}^{2+}$ release channel [56]. However, it is unclear how loss of PINK1 affects the activity of the mitochondrial $\mathrm{Na}^{+} / \mathrm{Ca}^{2+}$ exchanger. A direct regulation of the mitochondrial $\mathrm{Na}$ ${ }^{+} / \mathrm{Ca}^{2+}$ exchanger by PINK1-mediated phosphorylation is possible but difficult to demonstrate, since the molecular nature of the $\mathrm{Na}^{+} / \mathrm{Ca}^{2+}$ exchanger is unknown.

Mitochondrial dysfunction has long been thought to play an important role in the pathogenesis of Parkinson's disease [57]. This is based on earlier studies using postmortem idiopathic PD brains showing mitochondrial respiration impairment and oxidative damage [3], and on findings that mitochondrial complex I inhibitors, such as MPTP and rotenone, produce parkinsonian syndromes in humans and experimental animal models $[4,5]$. Our prior reports showing mitochondrial respiration defects in Parkin-/- and PINK1-/- mouse brains and linking these recessive PD genes to mitochondrial function provided experimental evidence in support of a causal role of mitochondrial functional impairment in PD pathogenesis [18,58]. The current study highlights the importance of mitochondrial permeability transition pore opening in PINK1 mediated mitochondrial respiration and function. Our recent unpublished work further showed that loss of Parkin or DJ-1 also leads to increases in mPTP opening (EG and JS, unpublished data). Thus, increased MPTP may be a common mechanism leading to PD pathogenesis.

In summary, our study highlights an important role of PINK1 in the regulation of mitochondrial permeability transition pore. Our findings suggest that dysregulation of the opening of the mPTP likely underlies impairment of mitochondrial respiration and reduction of mitochondrial transmembrane potential. Future studies will be needed to elucidate the mechanism by which PINK1 regulates mitochondrial calcium homeostasis and opening of the MPTP. Given the importance of mPTP opening in the regulation of the release of proapoptotic factors from mitochondria to the cytosol, it will be important to determine whether alteration of MPTP opening is a key mechanism underlying increased vulnerability of PINK1deficient cells to exogenous stressors $[11,59]$. In addition, future studies are needed to determine whether increased opening of the $\mathrm{mPTP}$ is a feature common to other genetic forms of the disease, and whether modulation of its opening may provide a novel therapeutic strategy for the treatment of Parkinson's disease.

\section{Competing interests}

The authors declare that they have no competing interests. 


\section{Acknowledgements}

We thank Hiroo Yamaguchi and other Shen lab members for discussions. This work was supported by a grant from the NINDS (R01 NS041779).

\section{Author details}

Center for Neurologic Diseases, Department of Neurology, Brigham \& Women's Hospital, Program in Neuroscience, Harvard Medical School, Boston, MA 02115, USA. Instituto de Biología y Genética Molecular, Universidad de Valladolid and Consejo Superior de Investigaciones Cientificas, Valladolid, Spain. ${ }^{3}$ Division of Biology and Howard Hughes Medical Institute, California Institute of Technology, Pasadena, CA 91125, USA. ${ }^{4}$ Université Pierre et Marie Curie-Paris 6, Centre de Recherche de I'Institut du Cerveau et de la Moelle épinière, UMR-S975, Paris, 75013, France.

\section{Authors' contribution}

CG and JS conceived and designed the study and wrote the manuscript. CG and EG carried out the experiments and obtained the data for Figures 1-9. LM, EC and CV participated in experimental design for Figure 9, and ZS and DC carried out the mitochondrial morphological analysis using the retrovira vectors mt-DsRed in Figure 8. All authors read and approved the final manuscript.

Received: 16 March 2012 Accepted: 25 May 2012

Published: 25 May 2012

\section{References}

1. Valente EM, Abou-Sleiman PM, Caputo V, Muqit MMK, Harvey K, Gispert S, Ali Z, Del Turco D, Bentivoglio AR, Healy DG, Albanese A, Nussbaum R, Gonzalez-Maldonado R, Deller T, Salvi S, Cortelli P, Gilks WP, Latchman DS, Harvey RJ, Dallapiccola B, Auburger G, Wood NW: Hereditary Early-Onset Parkinson's Disease Caused by Mutations in PINK1. Science 2004, 304:1158-1160.

2. Zhou C, Huang Y, Shao Y, May J, Prou D, Perier C, Dauer W, Schon EA, Przedborski S: The kinase domain of mitochondrial PINK1 faces the cytoplasm. Proc Natl Acad Sci U S A 2008, 105:12022-12027.

3. Schapira AH, Cooper JM, Dexter D, Jenner P, Clark JB, Marsden CD: Mitochondrial complex I deficiency in Parkinson's disease. Lancet 1989, 1:1269.

4. Langston JW, Ballard P, Tetrud JW, Irwin I: Chronic Parkinsonism in humans due to a product of meperidine-analog synthesis. Science 1983, 219:979-980.

5. Betarbet R, Sherer TB, MacKenzie G, Garcia-Osuna M, Panov AV, Greenamyre JT: Chronic systemic pesticide exposure reproduces features of Parkinson's disease. Nat Neurosci 2000, 3:1301-1306.

6. Park J, Lee SB, Lee S, Kim Y, Song S, Kim S, Bae E, Kim J, Shong M, Kim JM, Chung J: Mitochondrial dysfunction in Drosophila PINK1 mutants is complemented by parkin. Nature 2006, 441:1157-1161.

7. Clark IE, Dodson MW, Jiang C, Cao JH, Huh JR, Seol JH, Yoo SJ, Hay BA, Guo $\mathrm{M}$ : Drosophila pink1 is required for mitochondrial function and interacts genetically with parkin. Nature 2006, 441:1162-1166.

8. Poole AC, Thomas RE, Yu S, Vincow ES, Pallanck L: The mitochondrial fusion-promoting factor mitofusin is a substrate of the PINK1/parkin pathway. PLoS One 2010, 5:e10054

9. Deng H, Dodson MW, Huang H, Guo M: The Parkinson's disease genes pink1 and parkin promote mitochondrial fission and/or inhibit fusion in Drosophila. Proc Natl Acad Sci U S A 2008, 105:14503-14508.

10. Exner N, Treske B, Paquet D, Holmstrom K, Schiesling C, Gispert S, CarballoCarbajal I, Berg D, Hoepken HH, Gasser T, Kruger R, Winklhofer KF, Vogel F, Reichert AS, Auburger G, Kahle PJ, Schmid B, Haass C: Loss-of-function of human PINK1 results in mitochondrial pathology and can be rescued by parkin. J Neurosci 2007, 27:12413-12418,

11. Morais VA, Verstreken $P$, Roethig A, Smet J, Snellinx A, Vanbrabant M, Haddad D, Frezza C, Mandemakers W, Vogt-Weisenhorn D, Van Coster R, Wurst W, Scorrano L, De Strooper B: Parkinson's disease mutations in PINK1 result in decreased Complex I activity and deficient synaptic function. EMBO Mol Med 2009, 1:99-111.

12. Dagda RK, Cherra SJ 3rd, Kulich SM, Tandon A, Park D, Chu CT: Loss of PINK1 function promotes mitophagy through effects on oxidative stress and mitochondrial fission. J Biol Chem 2009, 284:13843-13855.
13. Lutz AK, Exner N, Fett ME, Schlehe JS, Kloos K, Lammermann K, Brunner B, Kurz-Drexler A, Vogel F, Reichert AS, Bouman L, Vogt-Weisenhorn D, Wurst W, Tatzelt J, Haass C, Winklhofer KF: Loss of parkin or PINK1 function increases Drp1-dependent mitochondrial fragmentation. J Biol Chem 2009, 284:22938-22951.

14. Yu W, Sun Y, Guo S, Lu B: The PINK1/Parkin pathway regulates mitochondrial dynamics and function in mammalian hippocampal and dopaminergic neurons. Hum Mol Genet 2011, 20:3227-3240.

15. Gandhi S, Wood-Kaczmar A, Yao Z, Plun-Favreau H, Deas E, Klupsch K, Downward J, Latchman DS, Tabrizi SJ, Wood NW, Duchen MR, Abramov AY: PINK1-associated Parkinson's disease is caused by neuronal vulnerability to calcium-induced cell death. Mol Cell 2009, 33:627-638.

16. Amo T, Sato S, Saiki S, Wolf AM, Toyomizu M, Gautier CA, Shen J, Ohta S, Hattori N: Mitochondrial membrane potential decrease caused by loss of PINK1 is not due to proton leak, but to respiratory chain defects. Neurobiol Dis 2010, 41:111-118.

17. Piccoli C, Sardanelli A, Scrima R, Ripoli M, Quarato G, D'Aprile A, Bellomo F, Scacco S, De Michele G, Filla A, luso A, Boffoli D, Capitanio N, Papa S: Mitochondrial respiratory dysfunction in familiar parkinsonism associated with PINK1 mutation. Neurochem Res 2008, 33:2565-2574.

18. Gautier CA, Kitada T, Shen J: Loss of PINK1 causes mitochondrial functional defects and increased sensitivity to oxidative stress. Proc Natl Acad Sci U S A 2008, 105:11364-11369.

19. Gegg ME, Cooper JM, Schapira AH, Taanman JW: Silencing of PINK1 expression affects mitochondrial DNA and oxidative phosphorylation in dopaminergic cells. PLoS One 2009, 4:e4756.

20. Gispert S, Ricciardi F, Kurz A, Azizov M, Hoepken HH, Becker D, Voos W, Leuner K, Muller WE, Kudin AP, Kunz WS, Zimmermann A, Roeper J, Wenzel D, Jendrach M, Garcia-Arencibia M, Fernandez-Ruiz J, Huber L, Rohrer H, Barrera M, Reichert AS, Rub U, Chen A, Nussbaum RL, Auburger G: Parkinson phenotype in aged PINK1-deficient mice is accompanied by progressive mitochondrial dysfunction in absence of neurodegeneration. PLOS ONE 2009, 4:e5777.

21. Abramov AY, Gegg M, Grunewald A, Wood NW, Klein C, Schapira AH: Bioenergetic consequences of PINK1 mutations in Parkinson disease. PLoS One 2011, 6:e25622.

22. Watanabe H, Smith MJ, Heilig E, Beglopoulos V, Kelleher RJ 3rd, Shen J: Indirect regulation of presenilins in CREB-mediated transcription. J Biol Chem 2009, 284:13705-13713.

23. Frezza C, Cipolat S, Scorrano L: Organelle isolation: functional mitochondria from mouse liver, muscle and cultured fibroblasts. Nat Protoc 2007, 2:287-295.

24. Petronilli V, Miotto G, Canton M, Brini M, Colonna R, Bernardi P, Di Lisa F: Transient and long-lasting openings of the mitochondrial permeability transition pore can be monitored directly in intact cells by changes in mitochondrial calcein fluorescence. Biophys J 1999, 76:725-734.

25. Chen H, Detmer SA, Ewald AJ, Griffin EE, Fraser SE, Chan DC: Mitofusins Mfn1 and Mfn2 coordinately regulate mitochondrial fusion and are essential for embryonic development. J Cell Biol 2003, 160:189-200.

26. De Vos KJ, Sheetz MP: Visualization and quantification of mitochondrial dynamics in living animal cells. Methods Cell Biol 2007, 80:627-682.

27. Zhang C, Wu B, Beglopoulos V, Wines-Samuelson M, Zhang D, Dragatsis I, Sudhof TC, Shen J: Presenilins are essential for regulating neurotransmitter release. Nature 2009, 460:632-636.

28. Peng M, Falk MJ, Haase VH, King R, Polyak E, Selak M, Yudkoff M, Hancock WW, Meade R, Saiki R, Lunceford AL, Clarke CF, Gasser DL: Primary coenzyme $\mathrm{Q}$ deficiency in Pdss2 mutant mice causes isolated renal disease. PLOS Genet 2008, 4:e1000061.

29. Ichas F, Mazat JP: From calcium signaling to cell death: two conformations for the mitochondrial permeability transition pore. Switching from low- to high-conductance state. Biochim Biophys Acta 1998, 1366:33-50

30. Petronilli V, Miotto G, Canton M, Colonna R, Bernardi P, Di Lisa F: Imaging the mitochondrial permeability transition pore in intact cells. Biofactors 1998, 8:263-272.

31. Bernardi P, Scorrano L, Colonna R, Petronilli V, Di Lisa F: Mitochondria and cell death. Mechanistic aspects and methodological issues. Eur I Biochem 1999, 264:687-701.

32. Huser J, Rechenmacher CE, Blatter LA: Imaging the permeability pore transition in single mitochondria. Biophys J 1998, 74:2129-2137. 
33. Gillessen T, Grasshoff C, Szinicz L: Mitochondrial permeability transition can be directly monitored in living neurons. Biomed Pharmacother 2002, 56:186-193.

34. Halestrap AP, Davidson AM: Inhibition of Ca2(+)-induced large-amplitude swelling of liver and heart mitochondria by cyclosporin is probably caused by the inhibitor binding to mitochondrial-matrix peptidyl-prolyl cis-trans isomerase and preventing it interacting with the adenine nucleotide translocase. Biochem J 1990, 268:153-160.

35. Liu J, Farmer JD Jr, Lane WS, Friedman J, Weissman I, Schreiber SL: Calcineurin is a common target of cyclophilin-cyclosporin A and FKBPFK506 complexes. Cell 1991, 66:807-815.

36. Henderson PJ, Lardy HA: Bongkrekic acid. An inhibitor of the adenine nucleotide translocase of mitochondria. J Biol Chem 1970, 245:1319-1326.

37. Friberg $H$, Ferrand-Drake M, Bengtsson F, Halestrap AP, Wieloch T: Cyclosporin A, but not FK 506, protects mitochondria and neurons against hypoglycemic damage and implicates the mitochondrial permeability transition in cell death. J Neurosci 1998, 18:5151-5159.

38. Yorimitsu M, Muranaka S, Sato EF, Fujita H, Abe K, Yasuda T, Inoue M, Utsumi K: Role of alpha-tocopherol in the regulation of mitochondrial permeability transition. Physiol Chem Phys Med NMR 2004, 36:95-107.

39. Reid AB, Kurten RC, McCullough SS, Brock RW, Hinson JA: Mechanisms of acetaminophen-induced hepatotoxicity: role of oxidative stress and mitochondrial permeability transition in freshly isolated mouse hepatocytes. J Pharmacol Exp Ther 2005, 312:509-516.

40. Poole AC, Thomas RE, Andrews LA, McBride HM, Whitworth AJ, Pallanck L: The PINK1/Parkin pathway regulates mitochondrial morphology. Proc Natl Acad Sci U S A 2008, 105:1638-1643.

41. Yang Y, Ouyang Y, Yang L, Beal MF, McQuibban A, Vogel H, Lu B: Pink1 regulates mitochondrial dynamics through interaction with the fission/ fusion machinery. Proc Natl Acad Sci U S A 2008, 105:7070-7075.

42. Sandebring A, Thomas KJ, Beilina A, van der Brug M, Cleland MM, Ahmad R, Miller DW, Zambrano I, Cowburn RF, Behbahani H, Cedazo-Minguez A, Cookson MR: Mitochondrial alterations in PINK1 deficient cells are influenced by calcineurin-dependent dephosphorylation of dynaminrelated protein 1. PLOS ONE 2009, 4:e5701.

43. Odagiri K, Katoh H, Kawashima H, Tanaka T, Ohtani H, Saotome M, Urushida $\mathrm{T}$, Satoh $\mathrm{H}$, Hayashi $\mathrm{H}$ : Local control of mitochondrial membrane potential, permeability transition pore and reactive oxygen species by calcium and calmodulin in rat ventricular myocytes. J Mol Cell Cardiol 2009, 46:989-997.

44. Shim JH, Yoon SH, Kim KH, Han JY, Ha JY, Hyun DH, Paek SH, Kang UJ, Zhuang $\mathrm{X}$, Son JH: The antioxidant Trolox helps recovery from the familial Parkinson's disease-specific mitochondrial deficits caused by PINK1- and DJ-1-deficiency in dopaminergic neuronal cells. Mitochondrion 2011, 11:707-715.

45. Fontaine E, Eriksson O, Ichas F, Bernardi P: Regulation of the permeability transition pore in skeletal muscle mitochondria. Modulation By electron flow through the respiratory chain complex i. J Biol Chem 1998, 273:1266212668.

46. Dumas JF, Argaud L, Cottet-Rousselle C, Vial G, Gonzalez C, Detaille D, Leverve $X$, Fontaine E: Effect of transient and permanent permeability transition pore opening on $\mathrm{NAD}(\mathrm{P}) \mathrm{H}$ localization in intact cells. $\mathrm{J} \mathrm{BiOl}$ Chem 2009, 284:15117-15125.

47. Moreira PI, Santos MS, Moreno A, Rego AC, Oliveira C: Effect of amyloid beta-peptide on permeability transition pore: a comparative study. J Neurosci Res 2002, 69:257-267.

48. Sharov VG, Todor A, Khanal S, Imai M, Sabbah HN: Cyclosporine A attenuates mitochondrial permeability transition and improves mitochondrial respiratory function in cardiomyocytes isolated from dogs with heart failure. J Mol Cell Cardiol 2007, 42:150-158.

49. Liu W, Vives-Bauza C, Acin-Perez R, Yamamoto A, Tan Y, Li Y, Magrane J, Stavarache MA, Shaffer S, Chang S, Kaplitt MG, Huang XY, Beal MF, Manfredi G, Li C: PINK1 defect causes mitochondrial dysfunction, proteasomal deficit and alpha-synuclein aggregation in cell culture models of Parkinson's disease. PLoS One 2009, 4:e4597.

50. Heeman B, Van den Haute C, Aelvoet SA, Valsecchi F, Rodenburg RJ, Reumers V, Debyser Z, Callewaert G, Koopman WJ, Willems PH, Baekelandt $\checkmark$ : Depletion of PINK1 affects mitochondrial metabolism, calcium homeostasis and energy maintenance. J Cell Sci 2011, 124:1115-1125.

51. Maj MC, Tkachyova I, Patel P, Addis JB, Mackay N, Levandovskiy V, Lee J,

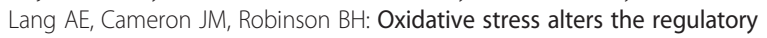

control of p66Shc and Akt in PINK1 deficient cells. Biochem Biophys Res Commun 2010, 399:331-335.

52. Ichas F, Jouaville LS, Mazat JP: Mitochondria are excitable organelles capable of generating and conveying electrical and calcium signals. Cell 1997, 89:1145-1153.

53. Baumgartner HK, Gerasimenko JV, Thorne C, Ferdek P, Pozzan T, Tepikin AV, Petersen $\mathrm{OH}$, Sutton $\mathrm{R}$, Watson AJ, Gerasimenko OV: Calcium elevation in mitochondria is the main $\mathrm{Ca} 2+$ requirement for mitochondrial permeability transition pore (mPTP) opening. J Biol Chem 2009, 284:20796-20803.

54. Vergun O, Reynolds IJ: Distinct characteristics of $\mathrm{Ca}(2+)$-induced depolarization of isolated brain and liver mitochondria. Biochim Biophys Acta 2005, 1709:127-137.

55. Cox DA, Conforti L, Sperelakis N, Matlib MA: Selectivity of inhibition of Na (+)-Ca2+ exchange of heart mitochondria by benzothiazepine CGP37157. J Cardiovasc Pharmacol 1993, 21:595-599.

56. Huang $X$, Zhai D, Huang Y: Study on the relationship between calciuminduced calcium release from mitochondria and PTP opening. Mol Cell Biochem 2000, 213:29-35.

57. Schapira AH: Mitochondrial pathology in Parkinson's disease. Mt Sinai J Med 2011, 78:872-881

58. Palacino JJ, Sagi D, Goldberg MS, Krauss S, Motz C, Wacker M, Klose J, Shen $\mathrm{J}$ : Mitochondrial dysfunction and oxidative damage in parkin-deficient mice. J Biol Chem 2004, 279:18614-18622

59. Petit A, Kawarai T, Paitel E, Sanjo N, Maj M, Scheid M, Chen F, Gu Y, Hasegawa H, Salehi-Rad S, Wang L, Rogaeva E, Fraser P, Robinson B, St George-Hyslop P, Tandon A: Wild-type PINK1 prevents basal and induced neuronal apoptosis, a protective effect abrogated by Parkinson diseaserelated mutations. J Biol Chem 2005, 280:34025-34032

doi:10.1186/1750-1326-7-22

Cite this article as: Gautier et al:: Regulation of mitochondrial permeability transition pore by PINK1. Molecular Neurodegeneration 2012 7:22

\section{Submit your next manuscript to BioMed Central and take full advantage of:}

- Convenient online submission

- Thorough peer review

- No space constraints or color figure charges

- Immediate publication on acceptance

- Inclusion in PubMed, CAS, Scopus and Google Scholar

- Research which is freely available for redistribution
C BioMed Central 